\title{
Self-Diffusion in a Gas-Fluidized Bed of Fine Powder
}

\author{
Jose Manuel Valverde, Antonio Castellanos, and Miguel Angel Sanchez Quintanilla \\ Departamento de Electrónica y Electromagnetismo, Facultad de Fisica, Avenida Reina Mercedes s/n, 41012 Seville, Spain
}

(Received 15 November 2000)

\begin{abstract}
We have investigated the self-diffusion in a stable gas-fluidized bed of fine powder. Two regimes have been observed: for gas velocities $v_{g}$ above the minimum fluidization velocity $v_{m}$ and below a critical gas velocity $v_{c}$ smaller than the minimum bubbling velocity $v_{b}$ the powder does not mix. Experimental measurements show the existence of yield stresses in this regime which are responsible for the static behavior of the bed. For $v_{g}>v_{c}$ the yield stress vanishes; the bed behaves like a fluid and displays a diffusive dynamics. In this region we have found that the diffusion coefficient $D$ increases with gas velocity until the bed expansion approaches its maximum value.
\end{abstract}

DOI: 10.1103/PhysRevLett.86.3020

PACS numbers: 45.70.Mg, 47.55.Kf, 47.55.Mh

The diffusive mixing of granular materials was first studied in granular shear flows of noncohesive coarse grains [1]. An analogy was made between the fluctuating or random component of particle motions (which are in a stationary nonequilibrium state) and the random motion of molecules in a dense gas (which is in thermodynamical equilibrium), and an effective granular temperature was defined in terms of the ensemble average of the squared fluctuation velocity, $3 T=\left\langle\mathbf{v}^{2}\right\rangle-\langle\mathbf{v}\rangle^{2}$. Wildman et al. [2] showed that the kinetic theory predictions used to relate granular temperature to self-diffusion in granular gases were accurate to within $10 \%-20 \%$ for packing fractions up to 0.6 in vibrofluidized granular beds. Fine powders are capable of being fluidized by a gas flow being the agent of transfer of momentum between particles [3]. Disequilibria created during collisions due to particle surface irregularities and local fluctuations in the gas velocity field are expected to be responsible for a random motion of particles. Because of the random component of particle motion in the gas-fluidized bed, the particles could exhibit a diffusive motion similar to that found in dense gases. The concept of granular temperature was extended to gas-fluidized beds by Cody et al. [4] who derived the granular temperature of the particles at the wall of a gas-fluidized bed by measuring the acoustic noise due to random particle impact at the wall. They showed that the granular temperature depends strongly on the fluidizing gas velocity. In their experimental study they used noncohesive glass spheres with diameters from 60 to $600 \mu \mathrm{m}$. They found that above the minimum fluidization velocity the average granular temperature increased directly proportional to the square of the gas superficial velocity. They also found that granular temperature changed inversely proportional to the square of particle diameter. Menon and Durian [5] reported on measurements by diffusion-wave spectroscopy of velocity fluctuations in gas-fluidized beds of spherical glass beads of diameters 49, 96, and $194 \mu \mathrm{m}$. Surprisingly, they found no fluctuations in the interval of uniform fluidization. This finding led them to the conclusion that "the uniformly fluidized state was a completely static state." They argued that particles in the fluidized state are held by enduring contacts. Velocity fluctuations were initiated by the instability to bubbling. However, it must be noted that the interval of uniform fluidization shrinks to almost zero for the 96 and $194 \mu \mathrm{m}$ particle diameter materials (see Fig. 1 of Ref. [5]). And for the bed with particles of $49 \mu \mathrm{m}$ in diameter the interval of stable fluidization, although appreciable, was very short (gas velocity from 0.2 to $0.25 \mathrm{~cm} / \mathrm{s}$ ). Furthermore, the bed expansion in the interval of uniform fluidization is quite small (a maximum of $4 \%$ of the initial bed height). The same results also apply to the gasfluidized beds of Cody et al. (minimum particle size $60 \mu \mathrm{m}$ ) who did not report on this short interval of null fluctuations but found an increase of the fluctuation velocity in the uniform fluidized bed as particle size was reduced due to enhanced gas flow in the dense phase. Rietema [6] outlined that interparticle cohesive forces could stabilize the homogeneously fluidized bed. These contact forces were assumed to give an effective elastic modulus to the bed that stabilizes the system against small disturbances. In that state the bed would behave like a weak solid rather than a fluid. To corroborate his thesis he showed that when the homogeneously fluidized bed was tilted the bed surface remained stable because of the existence of a certain mechanical strength. Tsinontides and Jackson [7] found experimentally that the mechanism of stabilization in the fluidized regime was the presence of yield stresses in the particle assemblies which form the fluidized but nonbubbling bed. However, Foscolo and Gibilaro [8] rejected Rietema's ideas. They stated that particles were free floating in the fluidized bed and proposed a stability criterion based on the dependence of the gas-particle drag force on the free volume. Whether stresses in the uniform fluidized bed are carried out by particle contacts or by collisions is still a matter of strong controversy. The absence of velocity fluctuations in the uniform fluidized bed before the onset of bubbling found by Menon and Durian [5] gives support to Rietema's arguments. On the other hand, the work of Cody et al. [4], who measured particle fluctuations in uniformly fluidized 
beds, would support the collisional model. In this paper we try to clarify this apparent controversy.

We have concentrated on polymer particles with a volume average diameter $d_{p}=8.53 \mu \mathrm{m}$, a commercially available xerographic toner (Canon CLC700). According to the small particle size this powder could not be fluidized homogeneously due to the strong interparticle adhesion forces. However, the addition of flow conditioners which reduces the cohesion between grains makes it possible to drive this powder into a homogeneous fluidization phase exhibiting a considerable bed expansion (up to a free volume of $\simeq 0.85$, a $40 \%$ increase over the initial value). Additionally the interval of uniform fluidization is very wide (gas velocity from 0.1 to $4 \mathrm{~mm} / \mathrm{s}$ ), allowing us to study in more detail the bed structure in this state. The considerable enhancement of gas flow in the dense phase would lead one to predict, according to Cody et al.'s [4] experiments, a very high value of granular temperature. On the contrary, the measurements of Menon and Durian [5] would lead us to predict a static structure. We observe both regimes, the solidlike for gas velocities below a critical value and the fluidlike for velocities larger than this critical value. In this dynamic interval we show that the mixing can be modeled by a diffusive process. From the calculated self-diffusion coefficient we estimate the granular temperature on the assumption that the kinetic theory of dense gases can be applied. Our results are compared with others found in the literature.

We have used two batches of toner pigmented with the primary colors yellow and magenta. Particle density $\left(\rho_{p}=1199 \mathrm{~kg} / \mathrm{m}^{3}\right)$ was measured by using an AccuPyc 1330 pycnometer. Toner particles are based on a random copolymer and their volume average diameter is $8.53 \pm 2.53 \mu \mathrm{m}$. They are irregular in shape, since they are formed by a grinding process. The powder bed was contained in a rectangular container made of polycarbonate, $4 \mathrm{~cm}$ width and $10 \mathrm{~cm}$ in height with a porous, sintered metal filter base $(\sim 5 \mu \mathrm{m}$ pore size). Beds of different lengths, $L$, have been used $(7.6,10$, and $20 \mathrm{~cm})$ to consider possible boundary effects. The container was divided in two halves by a thin plate that could slide upward. Both sides of the container were filled with the same mass of toner, one side with magenta toner and the other with yellow toner. The total weight per unit area was fixed to $\sim 80 \mathrm{~Pa}$ for the whole set of experiments. Then, the powder was subjected to a controlled upward gas flow. Dry nitrogen, dispensed from a tank of compressed gas, was used in order to avoid problems associated with an increase in cohesion due to humidity. The gas flow was controlled by a mass flow controller in the range from zero to $2000 \mathrm{~cm}^{3} / \mathrm{min}$. The gas pressure drop across the bed is measured by a differential pressure transducer. The minimum fluidization velocity is $v_{m} \simeq 0.1 \mathrm{~mm} / \mathrm{s}$ and the uniform fluidized powder becomes unstable at a gas velocity $v_{b} \simeq 4 \mathrm{~mm} / \mathrm{s}$ (minimum bubbling velocity) for a free volume $\epsilon \simeq 0.85$. Then the mean bed height begins to decrease, with marked oscillations of the free surface as visible gas bubbles burst. In every run the bed was driven to the bubbling phase $\left(v_{g}>4 \mathrm{~mm} / \mathrm{s}\right)$. Then $v_{g}$ was reduced to a value $\boldsymbol{v}_{b}>\boldsymbol{v}_{g}>\boldsymbol{v}_{m}$ to put the bed in a homogeneously fluidized state. For each fluidization velocity we calculated the average free volume of the bed from the bed height measurement (see Fig. 1). Once the powder was fluidized the slider was carefully withdrawn. At this point the mixing process of both samples started. In order to get quantitative measurements of the concentration of both colors in the mixture we made use of the tendency of powder particles to adhere to a paper surface. A piece of white card paper of the width of half of the container was carefully lowered edgewise into the initially yellow side so as to cleave it perpendicular to the gas flow path. Then, on carefully withdrawing the card, it was found to carry a replica of the bulk of the mixed powder. By this sampling method we took samples in time of the initially yellow side. In order to take quantitative values of the concentration of particles of both colors in time from these samples we developed a preliminary calibration procedure. We prepared samples mixing both colors (magenta and yellow) in known concentrations ranging from $0 \%$ magenta to $50 \%$ magenta. Then we cleaved every mixture pattern with a white paper and digitized the resulting image. We separated the digital image in the four primary 8-bit channels cyan, magenta, yellow, and black and an image software gave us the average grey value (ranging from 0 to 255) of each channel. The difference between the average grey value of yellow and magenta channels $(y)$ could be fitted to a second order polynomial law, $y=0.0574 c^{2}-6.607 c+208.47$, where $c$ is the magenta toner concentration in percent. This experimental law allows us to obtain the average magenta concentration of any sample taken during the mixing process from the difference between the average grey level of the yellow channel and the average grey level of the magenta channel.

According to the mixing properties of the bed two clearly differentiated regimes were found. In the interval

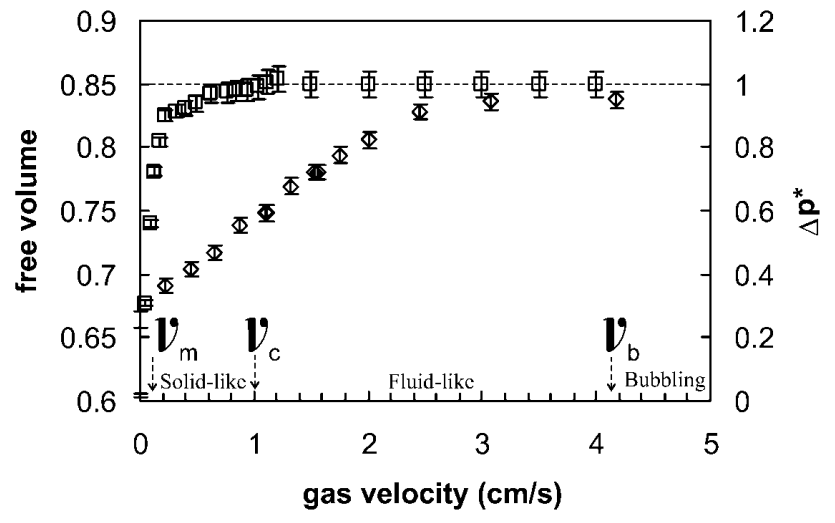

FIG. 1. Free volume $(\diamond)$ of the powder bed (a mixture of $50 \%$ magenta toner and $50 \%$ yellow toner) and gas pressure drop $\left(\Delta p^{*}\right)$ normalized to the bed weight per unit area $(\square)$ as a function of gas velocity. 
from $v_{g}=v_{m}=0.1 \mathrm{~mm} / \mathrm{s}$ to $v_{g}=v_{c}=1 \mathrm{~mm} / \mathrm{s}$ the powder did not mix; it remained in a stationary static state. This finding would be in agreement with Menon and Durian's [5] work who found no fluctuations in particle velocity. They claimed that the uniform fluidized bed would be in a static state due to stresses carried by particle permanent contacts. Indeed, we have observed that when the gas velocity $v_{g}$ is reduced from the initial value in the free bubbling range to a value $v_{m}<v_{g}<v_{c}$ the gas pressure drop lies below the weight of the bed (see Fig. 1); i.e., there is a fraction of the particles support that must derive from their mutual contact. Based on this we measured the tensile strength of uniform fluidized beds of xerographic toners. Details on these measurements are given elsewhere [9]. In Fig. 2 we represent experimental results obtained for the tensile strength, $\sigma_{t}$, of the Canon CLC700 toner. It is found that $\sigma_{t}$ depends on the free volume and decreases when the free volume is increased, i.e., when the fluidizing gas velocity is increased. We find that the tensile strength vanishes in our powders for a free volume $\epsilon_{c}$ between 0.72 (magenta) and 0.75 (yellow). Thus the fluidized powder bed would behave as a weak solid for a free volume $\epsilon<\epsilon_{c}$; i.e., it would be in a completely static state. From Fig. 1 we see that a free volume of the mixture between 0.72 and 0.75 corresponds to a gas velocity $v_{c} \simeq 1 \mathrm{~mm} / \mathrm{s}$ and, therefore, as we find experimentally, for $v_{g}<v_{c}$ no mixing is expected. The weak solidlike behavior of the fluidized bed for $\epsilon<\epsilon_{c}$ can also be inferred from settling experiments. When we come to measure the sedimentation rate of a homogeneously fluidized bed after gas supply is stopped we find that the head of the bed falls initially at a constant velocity, but when the bed reaches a free volume around 0.72 (in the case of magenta toner) the settling process slows down, indicating the formation of stresses carried by interparticle contacts that hinder sedimentation. A further evidence of the existence of a mechanical strength in the fluidized bed for $\epsilon<\epsilon_{c}$ can be obtained from tilting the fluidized bed. When the bed is slowly tilted it remains stable while the top surface is tilted as well. In Fig. 2

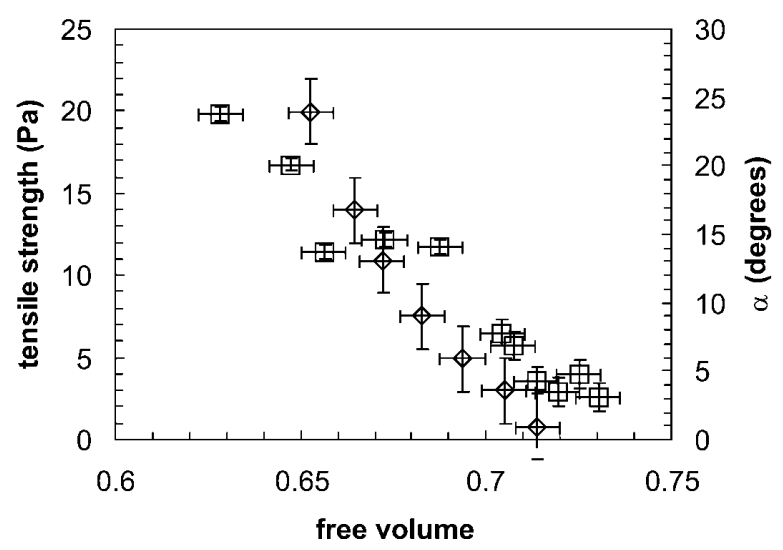

FIG. 2. Tensile strength $(\diamond)$ and angle of avalanche $(\square)$ as a function of the powder free volume for the magenta toner. the maximum stable angle is represented as a function of the fluidized bed free volume. We observe that this angle decreases as the tensile strength decreases and becomes zero when the tensile strength vanishes. At this point the bed behavior changes from solidlike to fluidlike.

In the second interval of uniform stable fluidization (gas velocity from 1 to $4 \mathrm{~mm} / \mathrm{s}$; free volume from 0.72 to 0.85 ), the whole bed weight is supported by the gas flow and the tensile strength is zero (see Fig. 2). The powder then takes on many of the properties of a fluid, its upper surface remaining horizontal when the container is tilted. There are not permanent contacts and stresses in this state are carried by collisions. Thus, the loss of mechanical strength cannot be claimed as the source of instability. In this regime the powder mixed successfully and the mixing rate was dependent on the gas velocity. Experimental values of magenta concentration in the initially yellow side are shown in Fig. 3 as a function of mixing time for different gas velocities. From this figure it is clear that as the gas velocity is increased the mixing process is fastened. If we decide to model the mixing process by a Fickian diffusion the flux of a component should be proportional to the diffusion coefficient and the concentration gradient of the constituent. In our special case of similar particles we can think of an effective self-diffusion coefficient $D$ which refers to the diffusion of toner particles relative to the rest. Under this assumption the mixing process could be described by means of a diffusion equation: $\partial c / \partial t=D \partial^{2} c / \partial x^{2}$, in which $x$ is the rectangular coordinate perpendicular to the separating plate along which mixing takes place and $c(x, t)$ is the concentration of magenta toner. The initial conditions are $c=0$ for $0<x<L / 2$ (initial yellow side), $c=0.5$ for $x=L / 2, c=1$ for $L / 2<x<L$ (initial magenta side). The boundary conditions are $d c / d x=0$ for $x=0$ and $x=L$. To compare with our experimental results we have to compute the average value of magenta toner concentration in the initial yellow side as a function of time, $\langle c\rangle(t)=2 / L \int_{0}^{L / 2} c(x, t) d x$. We have computed numerically the value of the diffusion coefficient $D$ for

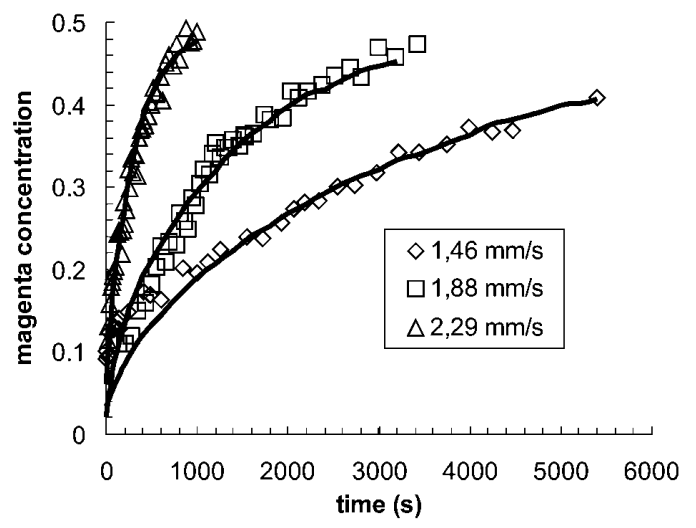

FIG. 3. Experimental values of the magenta toner concentration over the initially yellow side and theoretical curves assuming a Fickian diffusion process for different values of the gas velocity. 


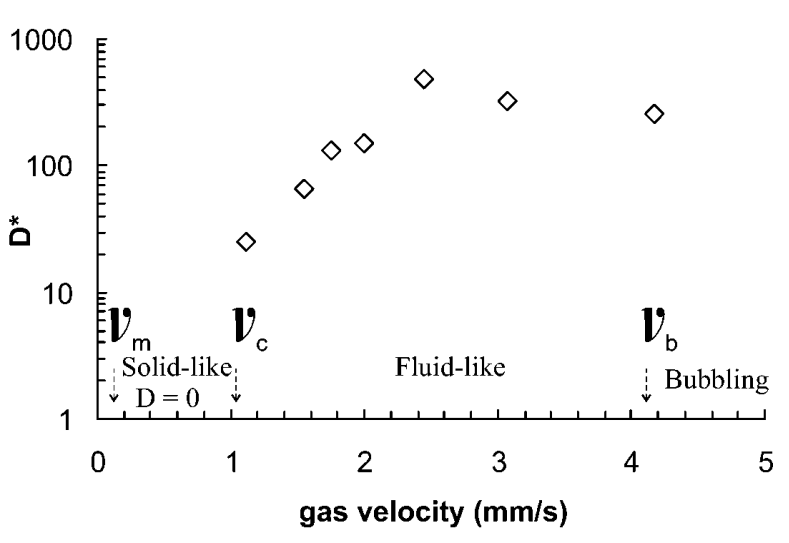

FIG. 4. Nondimensional diffusion coefficient $\left[D^{*}=D /\right.$ $\left.\left(v_{g} d_{p}\right)\right]$ obtained from the fit between experimental and theoretical values of magenta toner concentration as a function of gas velocity.

which $\sum_{i=0}^{i=N}\left[\langle c\rangle_{t}(i)-\langle c\rangle_{e}(i)\right]^{2}$ is minimum, $N$ being the total number of experimental points, $\langle c\rangle_{t}(i)$ the theoretical value, and $\langle c\rangle_{e}(i)$ the experimental value both at instant $i$. In Fig. 3 we plot the theoretical predictions for $\langle c\rangle(t)$ fitting in this way the experimental data. As can be seen from Fig. 3 theoretical curves from the diffusion model fit the experimental data pretty well. In Fig. 4 the values of $D$ obtained are represented as a function of the gas velocity. The results indicate that, in the range from 1 to $2 \mathrm{~mm} / \mathrm{s}, D$ increases exponentially with gas velocity. However, as the free volume approaches its maximum value $(\sim 0.85)$ the gas-solid contact is maximum and the mixing rate keeps constant and independent of gas flow. We now turn to the estimation of the granular temperature in the gas-fluidized bed from the obtained diffusion coefficients. According to the kinetic theory of dense gases, the diffusion constant of a hard spheres (particle diameter $d_{p}$ ) gas can be approximated by $D=d_{p} / 2 \sqrt{T}$, assuming a mean free path given by $d_{p} / 2$. In our case it is likely that particles aggregate due to the strong interparticle adhesive forces. However, this expression would allow us to have an estimation of the order of magnitude of the granular temperature. From our experimental values of the diffusion coefficient we estimate that the fluctuation velocity $\left(v_{f}=\sqrt{T}=2 D / d_{p}\right)$ increases exponentially with gas velocity up to a maximum which is 2 orders of magnitude larger than the gas velocity. As we have already stated the experimental measurements of Cody et al. [4] show a strong dependence of granular temperature on particle size. If we extrapolate their results to a particle size of $8.53 \mu \mathrm{m}$ (average diameter of toner particle) we would obtain a fluctuation velocity 2 orders of magnitude larger than gas velocity which is in agreement with our estimation.

In conclusion, up to now whether particles are free floating in the gas-fluidized bed or, on the contrary, are static has been a matter of strong debate. In most of the experiments the powders used to corroborate these arguments were composed of particles larger than $50 \mu \mathrm{m}$ to avoid interparticle adhesive forces. In that case the interval of uniform fluidization is very short and a detailed study of the bed structure is quite difficult. The use of fine particles with a reduced cohesivity because of the addition of flow conditioners has allowed us to reach a very wide interval of uniform fluidization where we have found two clearly differentiated regimes. For gas velocities below a critical value contact stresses persist and the bed has a nonvanishing yield stress that has been measured. In this interval particles are static and no mixing occurs. For gas velocities above the critical value and below the minimum bubbling velocity permanent contacts do not exist anymore, the yield stress becomes zero, and stresses are carried by collisions. This regime is characterized by a diffusive dynamics. The diffusion coefficient increases exponentially with gas velocity up to a maximum value corresponding to the maximum bed expansion point where gas-solid contact saturates. The granular temperature for the fully expanded bed, estimated based in the kinetic theory of dense gases, agrees in order of magnitude with the extrapolation of experimental data found in the literature for larger grains. The considerable bed expansion indicates an enhanced gas flow through the fluidized particles that is responsible for the very high values of the granular temperature estimated.

This research has been supported by Xerox Foundation and by the Spanish Government Agency Direccion General de Ciencia y Tecnologia (DGES) under Contract No. BMF2000-1056.

[1] V. V. R. Natarajan, M. L. Hunt, and E. D. Taylor, J. Fluid Mech. 304, 1 (1995).

[2] R. D. Wildman, J. M. Huntley, and J.P. Hansen, Phys. Rev. E 60, 7066 (1999).

[3] A. Castellanos, J. M. Valverde, A. T. Pérez, A. Ramos, and P. K. Watson, Phys. Rev. Lett. 82, 1156 (1999).

[4] G. D. Cody, D. J. Goldfarb, G. V. Storch, Jr., and A. N. Norris, Powder Technol. 87, 211 (1996).

[5] N. Menon and D. J. Durian, Phys. Rev. Lett. 79, 3407 (1997).

[6] K. Rietema, Chem. Eng. Sci. 28, 1493 (1973).

[7] S. C. Tsinontides and R. Jackson, J. Fluid Mech. 255, 237 (1993).

[8] P. U. Foscolo and L. G. Gibilaro, Chem. Eng. Sci. 39, 1667 (1984).

[9] J. M. Valverde, A. Castellanos, A. Ramos, A. T. Perez, M. A. Morgan, and P. K. Watson, Rev. Sci. Instrum. 71, 2791 (2000). 